\title{
Louis FOURNIER
}

Journaliste spécialisé dans le syndicalisme québécois

(1978)

\section{"Problèmes actuels}

\section{du mouvement syndical au Québec”}

Un document produit en version numérique par Jean-Marie Tremblay, bénévole, professeur de sociologie au Cégep de Chicoutimi

Courriel: jean-marie tremblay@uqac.ca

Site web pédagogique : http://www.uqac.ca/jmt-sociologue/

Dans le cadre de: "Les classiques des sciences sociales" Une bibliothèque numérique fondée et dirigée par Jean-Marie Tremblay, professeur de sociologie au Cégep de Chicoutimi Site web: http://classiques.uqac.ca/

Une collection développée en collaboration avec la Bibliothèque Paul-Émile-Boulet de l'Université du Québec à Chicoutimi Site web: http://bibliotheque.uqac.ca/ 
Cette édition électronique a été réalisée par Jean-Marie Tremblay, bénévole, professeur de sociologie au Cégep de Chicoutimi à partir de :

\section{Louis Fournier}

“Problèmes actuels du mouvement syndical au Québec”.

Un article publié dans la revue Politique aujourd'hui, no 7-8, 1978, pp. 129136. Numéro intitulé : “Québec : de l'indépendance au socialisme”.

[Autorisation formelle accordée par l'auteur le 30 novembre 2006 de diffuser cet article dans Les Classiques des sciences sociales.]

Courriel : louisfournier60@yahoo.ca

Polices de caractères utilisée :

Pour le texte: Times New Roman, 14 points.

Pour les citations : Times New Roman, 12 points.

Pour les notes de bas de page : Times New Roman, 12 points.

Édition électronique réalisée avec le traitement de textes Microsoft Word 2004 pour Macintosh.

Mise en page sur papier format : LETTRE (US letter), 8.5'’ x 11'’)

Édition numérique réalisée le 16 août 2007 à Chicoutimi, Ville de Saguenay, province de Québec, Canada. 


\section{Louis Fournier}

Journaliste spécialisé dans le syndicalisme québécois

\section{"Problèmes actuels du mouvement syndical au Québec”}

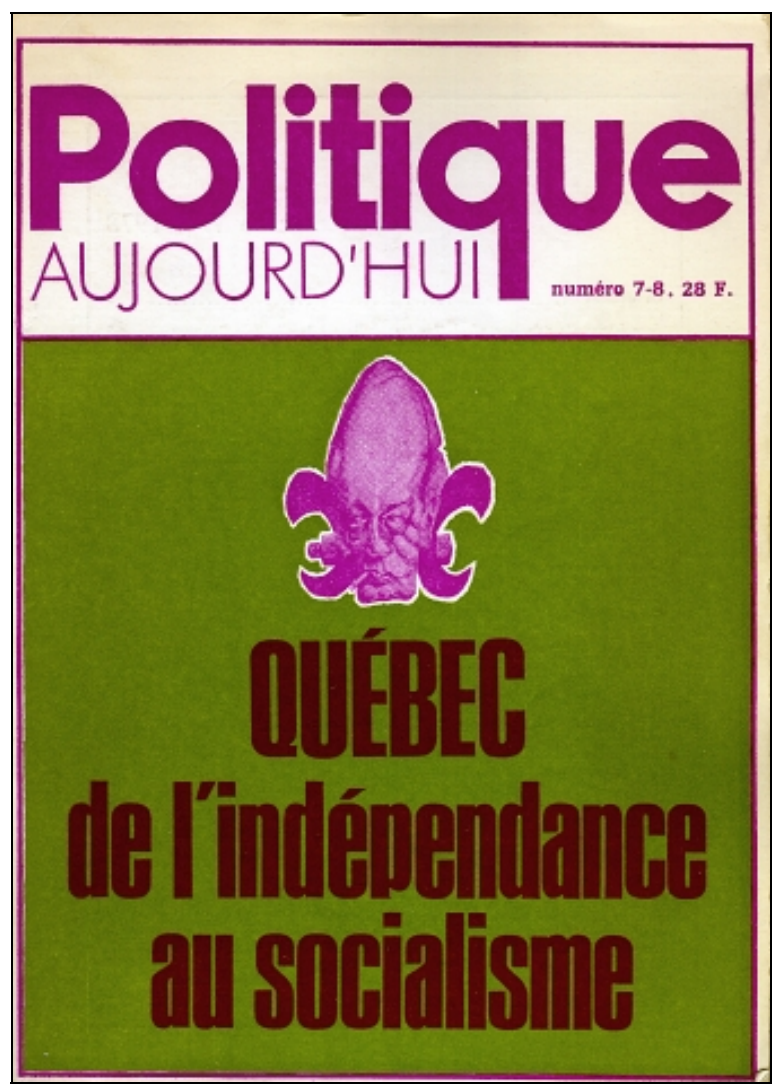

Un article publié dans la revue Politique aujourd'hui, no 7-8, 1978, pp. 129136. Numéro intitulé : “Québec : de l'indépendance au socialisme”. 


\section{Louis Fournier * \\ "Problèmes actuels du mouvement syndical au Québec".}

Un article publié dans la revue Politique aujourd'hui, no 7-8, 1978, pp. 129136. Numéro intitulé : “Québec : de l'indépendance au socialisme”.

La grande crise actuelle du capitalisme est au cœur des problèmes que doivent affronter les travailleurs du Québec et leurs organisations syndicales en 1978. C'est la toile de fond sur laquelle s'inscrivent les revendications et les luttes.

La crise économique, faut-il le rappeler, est sans précédent ici depuis la Grande Dépression des années 1930. L'hiver dernier, le taux de chômage a franchi le niveau-record de $12 \%$, frappant plus de 300.000 travailleurs dont près de la moitié ont moins de 25 ans.

Les licenciements et les fermetures d'entreprises se sont multipliés, particulièrement dans les secteurs industriels traditionnellement implantés au Québec comme le textile, le vêtement, la chaussure et le meuble - qui comptent pour 25\% des emplois de l'industrie manufacturière. La mise en chômage de dizaine de milliers de travailleurs a fait des coupes sombres dans les rangs des syndiqués.

En même temps, l'inflation persiste, sous la forme d'une flambée des prix qui brûle le pouvoir d'achat des travailleurs. Et ce d'autant plus que les salariés ont été les grandes victimes d'un programme qui a été le premier du genre, en temps de paix, au Canada et au Québec : le programme de contrôle des salaires et des prix. Il s'agit, essentielle-

* Journaliste spécialisé dans le mouvement syndical. 
ment, d'un " gel » des salaires, rigoureusement plafonnés et coupés lorsqu'ils outrepassaient les normes.

Ces « contrôles », décrétés en octobre 1975, n'ont été levés, partiellement, qu'en avril 1978. Ils ont concrétisé la pire offensive déclenchée contre les travailleurs et leurs organisations par le patronat et son principal « comité exécutif », soit le gouvernement central canadien.

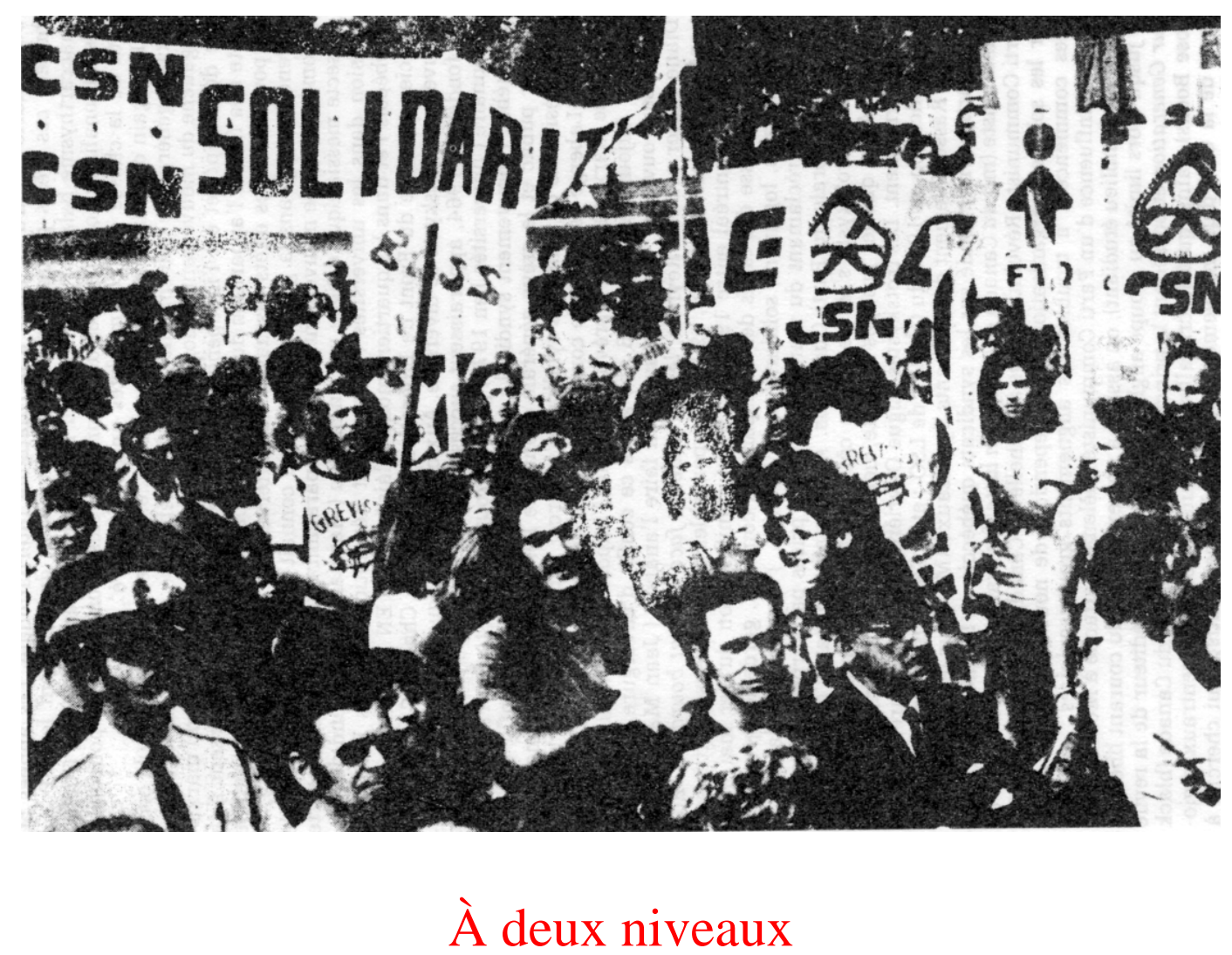

Les travailleurs québécois, rappelons-le, ont affaire à deux niveaux de gouvernement : le pouvoir central ou fédéral, à Ottawa, et le pouvoir provincial, à Québec. L'État bourgeois est ainsi divisé en deux tronçons, le plus important étant l'État fédéral qui détient les grands leviers d'action en matière économique. Or, c'est le gouvernement fédéral, celui du premier ministre Pierre-Elliott Trudeau, qui est à l'origine des fameux contrôles. 
La crise économique s'est maintenue, voire amplifiée depuis l'arrivée au pouvoir à Québec, en novembre 1976, du gouvernement provincial dirigé par le Parti Québécois ( $\mathrm{PQ}$, indépendantiste et réformiste) du premier ministre René Lévesque. Dès janvier 1977, le gouvernement Lévesque décidait cependant de mettre en veilleuse, à toutes fins utiles, la loi de contrôle des salaires dans les secteurs de juridiction provinciale (services publics, bâtiments, etc.).

En fait, les deux gouvernements, Ottawa et Québec, se rejettent mutuellement le blâme de la responsabilité de la crise. Cette petite guerre ne les empêche pas, somme toute, de gérer cette crise sur le dos des travailleurs.

\section{La défensive}

Au plan des luttes ouvrières, la crise et les "contrôles » ont eu pour effet de briser plusieurs mouvements revendicatifs, notamment la grève de quelque 40.000 travailleurs des pâtes et papiers, une industrie-clef au Québec. Le seul groupe de syndiqués qui ait réussi à défoncer les plafonds, en matière de " gel » des salaires, a été le Front commun de quelque 200.000 travailleurs des services publics de l'État québécois. Et ce, après une lutte acharnée qui a conduit le nouveau gouvernement du PQ à suspendre les « contrôles ».

Peu avant l'arrivée au pouvoir du PQ, les « contrôles » avaient aussi réussi à provoquer, le 14 octobre 1976, une grève générale de 24 heures qui a rallié 1.2 millions de syndiqués canadiens dont au-delà de 250.000 au Québec. C'était la première grève générale de cette ampleur dans l'histoire du Canada et en Amérique du Nord. Cette grèvefeu d'artifice n'a cependant pas eu de lendemains.

En réalité, la crise et les "contrôles » ont contribué à placer le mouvement syndical sur la défensive, en vue d'éviter tout recul. Les thèmes de lutte sont demeurés, depuis lors, la défense de l'emploi, la 
défense du pouvoir d'achat, la lutte contre les licenciements et les fermetures. Bref, la défense des acquis à conserver.

\section{Deux attitudes}

Le mouvement syndical, au Québec, est unanime dans son opposition au gouvernement fédéral du Canada et au Parti Libéral du premier ministre Trudeau. Il a refusé en bloc toute forme de concertation, de «tripartisme» (État-patronat-syndicats) avec ce gouvernement, considéré comme étant à la solde des multinationales et de l'impérialisme américain. Le mouvement ouvrier organisé a rejeté toute forme de « restrictions volontaires » qui auraient fait suite aux « contrôles ».

En revanche, le mouvement syndical a une attitude beaucoup plus ambiguë face au gouvernement du Québec, depuis la prise du pouvoir par le PQ le 15 novembre 1976.

Pour les syndicats, le Parti Québécois n'est pas un « parti des travailleurs », d'autant plus qu'il refuse toute forme de liens organiques avec le mouvement ouvrier, contrairement aux partis travaillistes ou sociaux-démocrates dans plusieurs pays. Néanmoins, le PQ se présente comme un parti « social-démocrate » et, certes, c'est la plus à gauche des formations sur l'échiquier politique québécois.

C'est pourquoi le gouvernement péquiste a réussi, informellement, à nouer une alliance tactique avec une large fraction du mouvement syndical. Il a notamment l'appui dit critique de la principale centrale syndicale, la Fédération des travailleurs du Québec, qui avait d'ailleurs appelé à voter pour le PQ.

Par contre, après une période d'espoir et d'attentisme, le mouvement d'opposition au gouvernement du PQ s'est affirmé, progressivement, au sein des deux autres grandes centrales syndicales : la Confé- 
dération des syndicats nationaux et la Centrale de l'enseignement du Québec.

Ces deux centrales sont d'ailleurs engagées dans des pourparlers d'unification qui les mettraient à égalité avec le FTQ, au plan des effectifs. Elles recrutent le gros de leur «membership » dans les secteurs public et parapublic alors que la centrale rivale, la FTQ, apparaît comme le bastion de la classe ouvrière dans le secteur privé.

\section{Des divergences}

L'opposition croissante de la CSN et de la CEQ au gouvernement Lévesque n'est pas étrangère, de toute évidence, à leur implantation massive dans les services publics. Elles doivent affronter l'État-patron, en l'occurrence le gouvernement du PQ. Les coupures de budget dans les réseaux de l'Éducation et des Affaires sociales laissent déjà présager des tensions majeures à

l'occasion de la prochaine grande ronde de négociation des conventions collectives. Ces conventions, d'une durée de trois ans, arrivent à échéance le 30 juin 1979. Et d'ici là, l'État patron entend bien redéfinir les « règles du jeu " pour casser la combativité des organisations syndicales dans ce secteur.

Face au gouvernement du Québec, les divergences au sein du mouvement syndical ne sont pas aussi tranchées qu'on peut imaginer, théoriquement, mais elles sont réelles. Il faut noter également que ces divergences existent non seulement entre la FTQ, d'une part, et la CSN-CEQ, d'autre part, mais aussi à l'intérieur de chacune des organisations. C'est ainsi qu'au sein de la FTQ, un fort courant s'oppose, par exemple, à toute politique de rapprochement un peu trop marqué avec le pouvoir. C'est le cas, entre autres, des militants du puissant syndicat canadien de la fonction publique (SCSP, 45.000 membres). 
L'un des principaux sujets de désaccord au sein du mouvement syndical a trait à l'ouverture dont il faut faire preuve à l'égard du gouvernement du PQ.

Officiellement, toutes les centrales affirment bien haut leur indépendance face à l'État et aussi le principe que les syndicats n'ont jamais arraché de gains que par leurs luttes, à la suite d'un rapport de forces fondé sur la mobilisation.

En pratique, la direction de la principale centrale, la FTQ, est plus sensible aux appels à la collaboration et à la concertation lancés par le Québec. Elle ne rejette pas à priori l'établissement d'un nouveau « contrat social » proposé par le PQ. Elle a accueilli d'un bon oeil la plupart des initiatives gouvernementales : réforme du Code du travail qui, entre autres, proscrit le recours aux briseurs de grève et favorise l'accès à la syndicalisation ; proclamation du français comme langue de travail ; projet de Charte sur la santé et la sécurité au travail ; projet de loi fixant des conditions minimales de travail pour les nonsyndiqués (60\% de la main-d'oeuvre) ; nationalisation partielle de l'industrie de l'amiante ; relèvement du salaire minimum (SMIG), etc.

En revanche, la CSN et la CEQ, tout en étant d'accord avec l'essentiel de ces réformes, sont beaucoup plus critiques face au pouvoir. Elles rappellent volontiers que le gouvernement Lévesque n'est pas - et ne se veut pas - un "gouvernement des travailleurs ». Le premier ministre René Lévesque ne s'en cache d'ailleurs pas, qui a déjà déclaré : « Le gouvernement ne doit rien ni aux centrales syndicales ni à quelque organisation patronale que ce soit. Il peut donc les traiter avec distance et avec respect ».

La CSN et la CEQ ont pris carrément leurs distances avec le pouvoir à l'occasion de la première expérience de « concertation » lancée par le gouvernement: le Sommet économique qui a réuni l'État, le patronat, le mouvement coopératif et les syndicats, en mai 1977. Alors que la FTQ s'est rendue à ce sommet à vive allure, les deux autres 
centrales n'y sont allées qu'après un large débat au cours duquel l'opposition à la " participation » et à la " collaboration de classes » s'est exprimée avec vigueur. L'influence au sein de la CSN et de la CEQ d'une gauche articulée, voire d'une extrême gauche parfois virulente, explique en partie cette situation.

\section{Des pratiques différentes}

Les pratiques syndicales des trois grandes centrales expliquent également leur comportement face au pouvoir.

La FTQ, qui se réclame de sa large base ouvrière et pro-PQ, pratique en général un syndicalisme de type nord-américain qu'elle qualifie de "pragmatique » et que ses adversaires ont étiqueté du nom de « syndicalisme d'affaires » (« business unionism » de tradition américaine). C'est un syndicalisme de type réformiste, qui vise à donner aux travailleurs leur part du gâteau capitaliste.

Du côté de la CSN et de la CEQ, on tente, de plus en plus, de pratiquer un syndicalisme dit de " combat » et "de classe ", illustrant le fait que les intérêts de la classe ouvrière et de la bourgeoisie sont irréconciliables en régime capitaliste. Ce syndicalisme plus "radical », surtout pratiqué par la CSN, n'est pas sans analogies avec les pratiques d'une organisation comme la Confédération française démocratique du travail (CFDT). Et, tout comme la CFDT, la CSN a été affectée par une scission à droite, en juin 1972, qui a donné naissance à la centrale des syndicats démocratiques (CSD, 50.000 membres) ressemblant étrangement à la Confédération française des travailleurs chrétiens (CFTC).

Les comparaisons en cette matière sont toujours boiteuses mais, grosso modo, on peut rapprocher les trois centrales québécoises de leurs vis-à-vis français de la façon suivante : la CSN est proche de la CFDT, la CEQ de la FEN et la FTQ de Force Ouvrière (FO). Au plan 
international, la FTQ et FO sont d'ailleurs affiliées à la Confédération internationale des syndicats libres (CISL) tandis que la CSN est membre de la Confédération mondiale du travail (CMT) - comme l'était la CFDT jusque tout récemment.

\section{L'unité syndicale}

Ce trop bref tour d'horizon des différences et des divergences, au sein du mouvement syndical, permet d'aborder l'une des questions les plus actuelles au Québec, celle de l'unité syndicale.

Cette unité répond à une aspiration profonde et ancienne du mouvement ouvrier. Les travailleurs portent en eux-mêmes l'instinct de l'unité, tout comme celui de leurs intérêts de classe. Au Québec, la multiplication des fronts communs intersyndicaux, depuis quelques années, va dans ce sens.

S'il est vrai que les principales organisations syndicales sont différentes, rivales et parfois même en divergences de vues et d'action, il est également vrai que le mouvement vers l'unité se manifeste fréquemment.

C'est ainsi que la convergence des positions de la CSN et de la CEQ leur ont permis d'entreprendre, en 1977, des pourparlers qui doivent mener, éventuellement, à l'unification. Les deux centrales ont mis sur pied un comité conjoint de l'unité syndicale et établi un échéancier à cette fin.

La démarche envisagée vise à « la mise en place d'une nouvelle organisation syndicale susceptible d'accueillir tous les travailleurs québécois partageant les mêmes orientations en matière d'action revendicative et la même volonté de solidarité ». En ce sens, la CSN et la CEQ ont invité tous les groupements syndicaux à s'associer à leur 
démarche car « le but de l'opération, c'est l'unité du mouvement syndical dans son ensemble ».

Cet appel réussira peut-être à rallier une partie des quelques 150 000 travailleurs québécois qui adhèrent à des syndicats indépendants (autonomes).

D'autres syndicats indépendants conserveront cependant leur statut, notamment ceux qui ont été expulsés du mouvement syndical bona fide, comme les Teamsters (routiers). Autrefois affiliés à la FTQ, les Teamsters forment le syndicat indépendant le plus puissant en Amérique du Nord (2,2 millions d'adhérents) et c'est celui dont la réputation est la plus douteuse. Quoi qu'il en soit, la création d'une nouvelle centrale, forte d'au moins 300.000 adhérents, changera profondément la configuration du mouvement syndical au Québec.

\section{La FTQ : un cas particulier}

On aura remarqué que la principale centrale, la FTQ, n'est pas associée aux démarches en vue de l'unité syndicale. Outre que certaines de ses positions politiques diffèrent de celles de ses rivales, la FTQ, a des structures fort particulières, en raison de ses liens organiques avec le mouvement syndical canadien et nord-américain. Cela n'est pas sans lui poser certains problèmes.

En fait, la FTQ n'est que l'aile québécoise du Congrès du travail du Canada (CTC), la grande centrale syndicale pancanadienne qui compte 2.3 millions d'adhérents. De plus, elle regroupe à la fois des syndicats canadiens et des Unions dites « internationales », c'est-à-dire canado-américaines et affiliées à l'AFL-CIO des États-Unis.

Le pouvoir, au sein de la FTQ, appartient en réalité aux Unions affiliées. L'adhésion à la centrale est d'ailleurs facultative et elle ne représente qu'environ les deux-tiers de son « membership » potentiel. 
Le défi majeur de la FTQ, c'est son émergence comme centrale authentiquement québécoise, ce qui l'entraîne dans des affrontements parfois très durs avec sa " maison-mère ", la CTC, et avec certaines Unions internationales. Depuis mai 1974, elle jouit d'un «statut spécial », plus autonome par rapport au CTC.

Cherchant à conquérir une plus grande indépendance encore vis-àvis du CTC la FTQ encourage également ses syndicats affiliés à affirmer leur autonomie face aux Unions « internationales » et même canadiennes. D'année en année, la liste s'allonge des syndicats qui ont rompu avec leur Union " américaine » pour former des syndicats distinctivement canadiens, voire exclusivement québécois.

La position de la FTQ est la suivante : les travailleurs québécois doivent se donner « le maximum d'autonomie » tout en demeurant, si c'est là leur décision, à l'intérieur de structures syndicales qui débordent les frontières du Québec. Le hic, c'est que cet "internationalisme » camoufle parfois une domination étrangère du syndicalisme québécois.

On peut en conclure qu'il sera impossible de créer une Centrale unique au Québec tant que la FTQ conservera son statut actuel.

\section{L'action politique ouvrière}

L'absence d'unité syndicale se manifeste également sur le plan de l'action politique ouvrière.

La FTQ donne un appui dit critique au Parti Québécois et, sur la scène fédérale, au Nouveau Parti Démocratique (NPD), le parti socialdémocrate canadien. 
De leur côté, la CSN et la CEQ ont lancé, auprès de leurs affiliés, un large débat sur les conditions de formation d'un véritable " parti des travailleurs » au Québec, en novembre 1976, contribuant ainsi à la victoire du PQ.

En effet, l'une des « originalités » de la scène politique québécoise, c'est qu'on n'y trouve aucun parti ouvrier digne de ce nom, ni socialiste ni communiste, mais des groupuscules et sectes sans influence réelle auprès des travailleurs. L'absence d'un tel parti ouvrier contribue d'ailleurs à la prolifération des organisations d'extrême-gauche de toutes tendances.

Chacune des trois grandes centrales syndicales est officiellement favorable à un socialisme " démocratique » et " québécois » mais aucune d'entre elles n'a de relais politique - si l'on excepte la FTQ qui appuie transitoirement le PQ.

La plupart des leaders syndicaux s'entendent sur la nécessité de construire un " parti de la classe ouvrière » au Québec. Cependant, les divergences surgissent dès qu'on envisage les conditions de sa formation, son opportunité dans la conjoncture actuelle, ses liens éventuels avec le mouvement syndical.

Il est clair que les syndicats ont un rôle à jouer dans la mise sur pied d'un parti car ils ont besoin d'un «bras » politique, ce à quoi le PQ se refuse. Cependant, les syndicats sont des organisations de masse et doivent le demeurer. Ils ne peuvent se laisser « déstabiliser » par ceux qui voudraient leur voir jouer un rôle de parti, ce qui n'est pas de leur ressort.

L'idée a été lancée de créer d'abord un " mouvement socialiste ", avant-garde d'un futur parti. Ce mouvement, large et unitaire, pourrait rassembler des courants socialistes qui, pour l'heure, sont très dispersés et disparates. Il mûrirait à même les luttes ouvrières et ferait aussi 
pression sur le PQ. Résultat net : il n'existe pas encore d'organisation politique autonome de la classe ouvrière au Québec.

\section{La question nationale}

L'une des raisons les plus fréquemment avancées pour expliquer ce phénomène est l'omniprésence de la question nationale au Québec, c'est-à-dire du statut des Québécois francophones par rapport au Canada.

La formation du Parti Québécois, en Octobre 1968, a eu des effets majeurs sur l'action politique ouvrière. Le PQ a rallié une large fraction de militants syndicaux qui soutiennent que seul un État Québécois souverain permettra de construire, dans une deuxième étape, le socialisme. Le Conseil central de Montréal (CSN) affirme que «l'indépendance nationale du Québec est indispensable à la prise du pouvoir par les travailleurs et au socialisme ».

Cette théorie des deux étapes - indépendance d'abord, socialisme ensuite - est de plus en plus contestée au sein du mouvement syndical, surtout à la CSN et à la CEQ. La lutte pour l'indépendance, affirme-ton, ne doit pas être confisquée (et détournée) par le PQ mais assumée par le mouvement ouvrier et ses organisations. Le défi est donc de jumeler le projet socialiste et le mouvement de libération nationale au Québec.

L'orientation du mouvement syndical, dans les années 1970, est devenue plus franchement anticapitaliste. Cette orientation doit s'insérer toutefois dans une problématique spécifique : la question nationale, les rapports Québec-Canada.

Or, jusqu'où le projet souverainiste du PQ coïncide-t-il avec les intérêts des travailleurs québécois? Question fondamentale que pose aussi, à sa façon, l'un des leaders syndicaux québécois parmi les plus 
prestigieux, Marcel Pepin, ancien président de la CSN et aujourd'hui président de la Confédération mondiale du travail :

« La question nationale, dit-il, est primordiale mais la question sociale ne peut être écartée. Les travailleurs des usines, des entreprises resteraient dans leurs mêmes difficultés, dans leurs mêmes souffrances, dans leur même aliénation si le Québec se contentait de régler la question nationale, fût-ce par l'indépendance, et si la condition ouvrière restait ce qu'elle est »...

\section{Fin du texte}

